\title{
The influence of heavy goods vehicle traffic on accidents on different types of Spanish interurban roads
}

\author{
B. Arenas Ramírez ，F. Aparicio Izquierdo ，C. González Fernández ～, A. Gómez Méndez \\ Automobile Research Institute (INSIA), Polytechnic University of Madrid (UPM), José Gutiérrez de Abascal N2, 28006 Madrid, Spain \\ Statistical Laboratory, Polytechnic University of Madrid (UPM), José Gutiérrez de Abascal N2, 28006 Madrid, Spain
}

\begin{abstract}
A B S T R A C T
This paper illustrates a methodology developed to analyze the influence of traffic conditions, i.e. volume and composition on accidents on different types of interurban roads in Spain, by applying negative binomial models. The annual average daily traffic was identified as the most important variable, followed by the percentage of heavy goods vehicles, and different covariate patterns were found for each road type. The analysis of hypothetical scenarios of the reduction of heavy goods vehicles in two of the most representative freight transportation corridors, combined with hypotheses of total daily traffic mean intensity variation, produced by the existence or absence of induced traffic gives rise to several scenarios. In all cases a reduction in the total number of accidents would occur as a result of the drop in the number of heavy goods transport vehicles, However the higher traffic intensity, resulting of the induction of other vehicular traffic, reduces the effects on the number of accidents on single carriageway road segments compared with high capacity roads, due to the increase in exposure. This type of analysis provides objective elements for evaluating policies that encourage modal shifts and road safety enhancements.
\end{abstract}

\section{Introduction}

In Spain, the internal transport of goods, as measured in ton-km and carried out by different modes, of transportation, has experienced a considerable increase since 1950 This growth has not been the same in all modes, with road transport having experienced the greatest increase in the period. The share of road transport increased from $24.21 \%$ in 1950 to $84.62 \%$ in 2006 , while rail transport decreased from $35.74 \%$ to $2.66 \%$ (Fig. 1). The increase in mobility and road freight traffic (in millions of vehiclekilometres) has highlighted the need for a quantitative evaluation of its influence on safety and the environment (Fig. 2). Between 1993 and 2005 the total number of accidents on Spanish interurban roads and those involving at least one HGV, have increased by $19 \%$ and $17 \%$, respectively (Fig. 3 ). On average, in $5.7 \%$ of accidents there was at least one HGV involved, $86 \%$ of which happened on interurban roads: $57 \%$ on single carriageway roads and $37 \%$ on high capacity roads and the remaining $5 \%$ on minor roads (data from 2005).
In the 2005-2008 Strategic Road Safety Plan

the General Directorate of Traffic set the general aim of improving road safety in Spain, and proposed reducing the number of deaths by $40 \%$ by 2008 , from the 2003 level as well as reducing the seriousness of accidents. Taking account of the country's accident pattern, the PESV05-08 set as one of its strategic objectives to reduce the total number of HGVs involved in accidents with victims on main roads and single carriageway roads.

The increasing trend in freight road traffic, mobility and accidents, are the motivations for this study, which is related to road transport and the evaluation of specific countermeasures devoted to increasing the safety performance of Spanish roads.

Bearing in mind the increasing trend in goods traffic, the increase in mobility on the Spanish roads and the greater severity of accidents involving HGVs on interurban roads, the interest in this study is evident. In this work, we propose a methodology to analyze the influence of HGVs on traffic accidents, using general linear models.

This paper first addresses the identification of factors related to vehicle flow and composition (i.e., cars and HGV vehicles), in road accidents on road sections of the state network (RCE), by using negative binomial count regression models to predict accidents. Then, the paper addresses the analysis and evaluation of hypothetical HGV reduction scenarios based on the decrease of HGVs annual average daily traffic on road sections of two representative freight transportation corridors. 


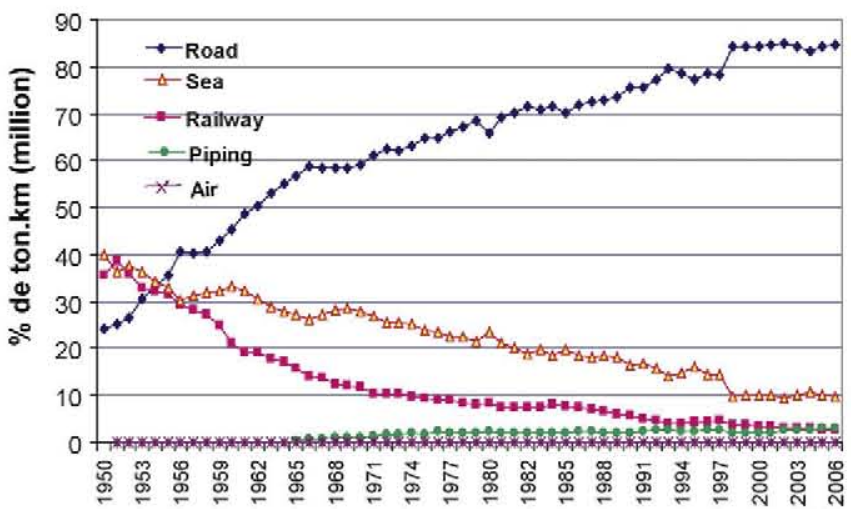

Fig. 1. Freight modal shift transport (in \% of ton-km (million)) 1950-2006.

The format of the paper is as follows: Section 2 briefly presents the literature review. Section 3 describes the data used in the work. In Section 4, some details of the model, the goodness of fit measures, and some ideas about the interpretation of the coefficients are given. Section 5 analyses the fitted model. In Section 6 the applications to the two freight transportation corridors are detailed, along with the study of hypothetical traffic scenarios in these two corridors. Section 7 summarizes main conclusions, and finally an Appendix A is included with the acronyms used in the paper.

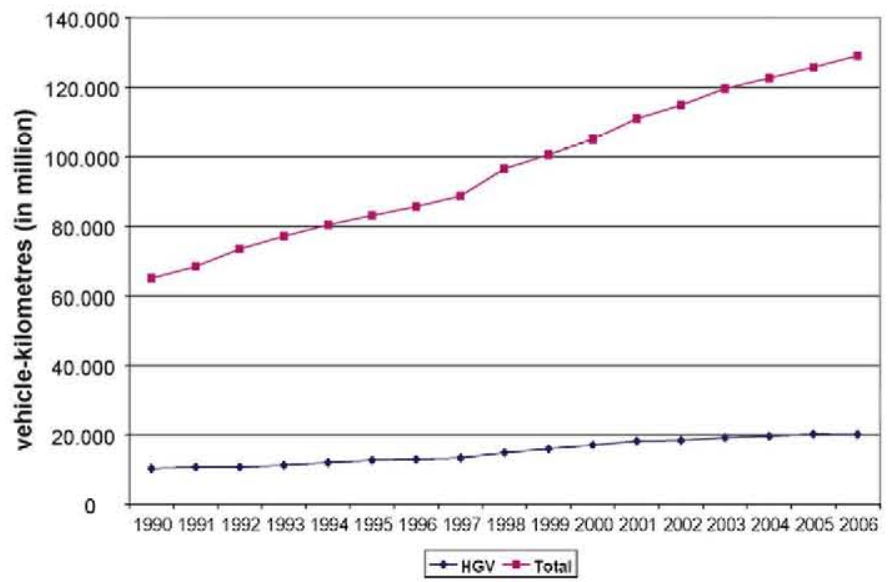

Fig. 2. Annual mobility (total and HGV) on Spanish RCE roads 1993-2005.

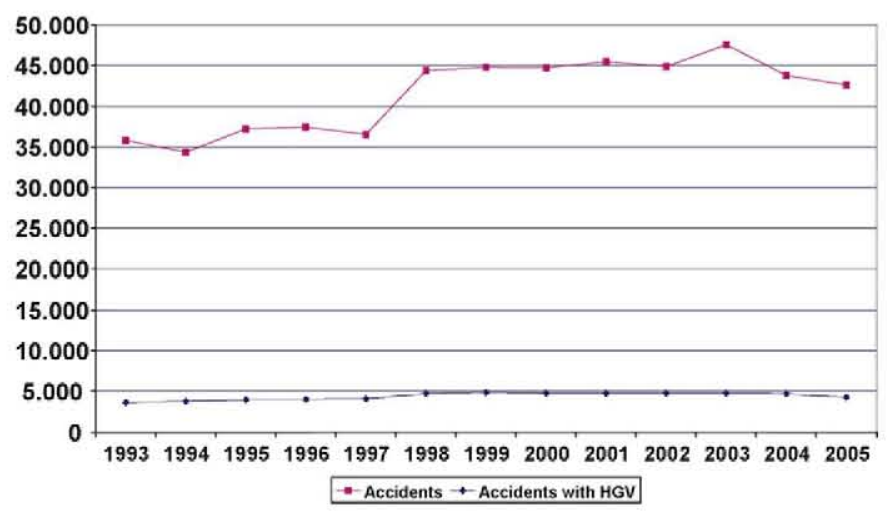

Fig. 3. Accidents figures on Spanish roads 1993-2005.

\section{Literature review}

Poisson and negative binomial regression models have been applied to estimate accident frequency and accident rates, with non-behavioural factors like road features, traffic characteristics, and weather or environmental conditions. From an empirical standpoint, the relation between crash frequency and vehicle-kilometres travelled and environmental conditions can be found in Jovanis and Chang (1986), with environmental factors in Shankar et al. (1995), with average hourly traffic volume per lane, average occupancy, lane occupation, average speed, and its standard deviation, curvature, and exposure in Garber and Wu (2001); and with road geometry and traffic characteristics in Vogt and Bared (1998), Miaou and Lum (1993), and Abdel-Aty and Radwan (2000).

Other studies have attempted to relate accident rates with traffic characteristics and the frequency of intersections (Ivan and O'Mara, 1997); hourly traffic volume (Martin, 2002); level of service, light conditions and the site accident characteristics (Ivan et al., 1999); with weather and light (Fridstrøm et al., 1995); and with the hourly traffic flow of cars and lorries (Hiselius, 2004).

Both approaches (Poisson and negative binomial regression) are considered appropriate from a statistical viewpoint because of the distribution of crash counts, but within this methodological framework, the negative binomial regression is preferred if overdispersion is observed, as is common in traffic accident data.

Few studies have studied the effect of heterogeneous flows, and particularly the effect that the presence of HGVs in the traffic flow has on accidents. The study most closely related to this paper is that by Hiselius (2004). In addition, few studies have used prediction models as tools for simulating and analysing new scenarios created by variations in traffic conditions. Precisely, in this work the accident prediction model was used to simulate and evaluate freight transport corridors under specific traffic conditions that include the reduction in the number of HGVs.

\section{Data}

This work used accident data from the DGT database (Accidents database, 2001) which covers police-reported accidents with at least one person injured during 2001 in segments belonging to different roads categories of the RCE network, i.e., toll motorways (AP), dual carriageways (AV), two undivided dual carriageways (DC), and single carriageway roads $(C)$.

The general criterion for including an accident in the database is that it is accident with victims and did not depend on the road type. Following this criterion, it may be thought that all accidents where there was a fatality or serious injuries are reported. However, in accidents with minor injuries there may be a certain loss of information, but this has still not been objectively evaluated, and is under discussion by several researchers (Lardelli-Claret et al., 2003). Segments refer here to bi-directional stretches, and can be considered to have homogeneous traffic conditions and constant traffic flow.

Traffic data are available for the whole RCE network in terms of aggregate estimates of average annual daily traffic (AADT), and average annual daily traffic per vehicle type, i.e., average annual HGV daily traffic (AADTHGV) in the traffic map produced by the Ministry of Public Works (Traffic Map, 2001). Traffic flow is counted as the number of vehicles through a fixed section in both directions, and the counts are performed using both portable counting instruments and permanent inductive loop detectors. Only 2541 road segments were extracted out of a total of 3085 from the 2001 traffic map, after selection criteria based on the complete information for traffic flow and reported accidents. 
Table 1

Road segment data.

\begin{tabular}{lcrc}
\hline Road type & \multicolumn{2}{l}{ Segments } & Length $(\mathrm{km})$ \\
\cline { 2 - 3 } & No. & \multicolumn{1}{c}{$\%$} \\
\hline AP & 154 & 6.06 & 1632 \\
AV & 692 & 27.23 & 5564 \\
DC & 188 & 7.40 & 349 \\
$C$ & 1507 & 59.31 & 14281 \\
Total & 2541 & 100.00 & 21886 \\
\hline
\end{tabular}

Table 1 lists the total number and length of the segments by road type, forming part of the RCE. In order to fit the models, the observations from the 2541 segments were used, of which $59.31 \%$ belong to single carriageway roads (C), with length of $14,281 \mathrm{~km}$, that is, $65.25 \%$ of the length of the state network studied. The 1034 segments of high capacity roads (AP, AV, and DC), are taken as a whole, form a total length of $7545 \mathrm{~km}$, which represents $34.47 \%$ of the total road network length studied. This assures that the two main groups of roads in the Spanish network are well represented.

More than $88 \%$ of traffic accidents in 2001 , were on single carriageway roads ( $C, 47.19 \%$ ) and on motorways (AV, $40.95 \%)$. These are two very different types of roads regarding their geometrical characteristics, traffic, and exposure.

Table 2 summarizes the global traffic data statistics by type of road for traffic variables and the exposure measure to be used in the models. The mean traffic intensity (AADT) is higher on AP, AV, and $D C$ than on single carriageway roads $(C)$, as a result of the greater capacity of the former since they have two or more lanes and a divided carriageway, one or more for each traffic direction.

The mean values of the percentage of heavy goods vehicles (\%HGVS) are very similar for the different road types. However, there is a wide range on single carriageway roads, which varies from a minimum of $1.875 \%$ to a maximum of $92.605 \%$. On AV and $\mathrm{DC}$ roads, the range and variance are lower than in single carriageway road, which is indicative of less heterogeneity in traffic flow, while on AP, roads, greater homogeneity is observed in comparison with the other categories.

Therefore, analysis of the traffic data reveals two main points: the very different traffic characteristics of high capacity roads and single carriageway roads, and the heterogeneity between segments of the same road type.

\section{Methodology}

The statistical models most commonly used to explain the relationship between motor vehicle accidents and a set of predictor variables, or regressors, are the Poisson and negative binomial regression models. In this work, the response variable is the expected number of accidents in a segment, and the potential regressors are both continuous (i.e., traffic flow, traffic composition) and categorical (i.e., type of segment).

In order to apply the results to segments with different traffic and exposures (measured in millions of vehicle-kilometres as $\mathrm{vk}_{j}=365 l_{j} \mathrm{AADT}_{j} / 10^{6}$, where $\mathrm{AADT}_{j}$ and $l_{j}$ are, respectively, the average annual daily traffic and length of the jth segment), and the accident rate of the $j$ th segment $\lambda_{j}$ is defined as the expected number of accidents, $E\left[Y_{j}\right]$, per million-vehicle $\mathrm{km}$. Therefore, the regression model is then expressed as $E\left[Y_{j}\right]=\lambda_{j} \mathrm{vk}_{j}$, using the loglink function to relate the response to the linear predictor as the log-link function is the natural or canonical link function. The general expression for the estimated model is then:

$\widehat{E\left[Y_{j}\right]}=\exp \left(\hat{\beta}_{0}+\sum_{m=1}^{M} \hat{\beta}_{m} X_{m j}+\hat{\beta}_{v} \ln \mathrm{vk}_{j}\right)$,

with the restriction $\hat{\beta}_{v}=1$.

If $Y_{j}$ is assumed to be a random variable with Poisson distribution, both its expected value and variance are equal. However, in practice, the variance tends to be larger than the mean, and so-called overdispersion occurs. If this is the case, the Poisson model is unlikely to be good and needs reappraisal, because the overdispersion does not affect the values of the model parameters, but can affect the Wald statistics or likelihood statistics for testing the hypothesis about the parameters. Thus, overdispersion is a problem if the objective is to identify the most relevant variables in modeling the relationships between accidents and the traffic and segment characteristics.

The negative binomial regression model accounts for the overdispersion and considers the heterogeneity of the segments that, even with the same covariate pattern, differ in their accident rate. Thus, the true accident rate in segments with a covariate pattern $X_{h}, \Lambda_{h}$ is considered to be $\Gamma(\kappa, a)$, being $E\left[\Lambda_{h}\right]=\kappa \cdot a$ and $\operatorname{Var}\left[\Lambda_{h}\right]=\kappa \cdot a^{2}$. So, if the number of accidents $Y_{h}$, is Poisson $\left(\Lambda_{h}\right)$, then the marginal distribution of $Y_{h}$ is a negative binomial with

Table 2

Summary statistics of traffic data. Global and per road type. Year 2001.

\begin{tabular}{|c|c|c|c|c|c|c|}
\hline Variable & Name & Sample & Minimum & Maximum & Mean & S.D. \\
\hline \multirow{5}{*}{ Average annual daily traffic } & \multirow{5}{*}{ AADT } & Global & 233 & 292589 & 17971.55 & 25109.77 \\
\hline & & AP & 5660 & 120626 & 27089 & 22092.20 \\
\hline & & AV & 1330 & 292589 & 34550 & 36617.60 \\
\hline & & DC & 1088 & 149153 & 29264 & 27211.80 \\
\hline & & C & 233 & 96701 & 8017 & 7535.24 \\
\hline \multirow{5}{*}{ Average annual HGV daily traffic } & \multirow{5}{*}{ AADTHGV } & Global & 8 & 30170 & 2556.04 & 3121.88 \\
\hline & & $\mathrm{AP}$ & 398 & 30170 & 4274.93 & 4872.78 \\
\hline & & AV & 412 & 21413 & 4790.71 & 3727.55 \\
\hline & & DC & 113 & 16577 & 3456.66 & 3378.58 \\
\hline & & C & 8 & 10893 & 1241.89 & 1344.14 \\
\hline \multirow{5}{*}{ Percentage HGVs } & \multirow{5}{*}{$\% \mathrm{HGV}$} & Global & 1.87 & 92.60 & 16.23 & 9.56 \\
\hline & & $\mathrm{AP}$ & 4.57 & 33.90 & 14.36 & 6.64 \\
\hline & & AV & 1.86 & 54.23 & 18.06 & 9.58 \\
\hline & & DC & 1.88 & 48.28 & 13.82 & 9.16 \\
\hline & & $C$ & 1.87 & 92.60 & 15.88 & 9.71 \\
\hline \multirow{5}{*}{$\begin{array}{l}\text { Total exposure in million of } \\
\text { vehicle-kilometres }\end{array}$} & \multirow{5}{*}{ vk } & Global & 0.03 & 776.66 & 40.88 & 60.88 \\
\hline & & AP & 0.89 & 361.85 & 86.98 & 68.04 \\
\hline & & AV & 0.80 & 776.66 & 80.19 & 90.09 \\
\hline & & DC & 0.06 & 274.53 & 22.19 & 35.11 \\
\hline & & $C$ & 0,03 & 197.98 & 20.45 & 22.69 \\
\hline
\end{tabular}


Table 3

Negative binomial model for accidents.

\begin{tabular}{|c|c|c|c|c|}
\hline$X_{i}$ & $\hat{\beta}[\mathrm{Cl}-95 \%]$ & IRR [CI-95\%] & $t$-Statistic & ( $p$-value) \\
\hline $\ln (\mathrm{AADT})$ & $-0.1111[-0.153 ;-0.069]$ & $0.895[0.858 ; 0.933]$ & -5.241 & $(<0.0001)$ \\
\hline $\ln (\% \mathrm{HGV})$ & $-0.1639[-0.245 ;-0.083]$ & $0.849[0.782 ; 0.920]$ & -3.959 & $(<0.0001)$ \\
\hline \multicolumn{5}{|l|}{ Road } \\
\hline $\mathrm{AP}$ & $-1.5115[-2.295 ;-0.729]$ & $0.220[0.100 ; 0.483]$ & -3.783 & $(0.0002)$ \\
\hline AV & $-0.9835[-1.372 ;-0.595]$ & $0.374[0.253 ; 0.552]$ & -4.957 & $(<0.0001)$ \\
\hline DC & $-1.2927[-1.895 ;-0.690]$ & $0.274[0.150 ; 0.501]$ & -4.204 & $(<0.0001)$ \\
\hline \multicolumn{5}{|l|}{$\ln (\% \mathrm{HGV})_{i}$} \\
\hline $\mathrm{AP}$ & $0.2277[-0.070 ; 0.525]$ & $1.066[0.932 ; 1.691]$ & 1.498 & $(0.1341)$ \\
\hline AV & $0.1742[0.04 ; 0.308]$ & $1.010[1.041 ; 1.361]$ & 2.543 & $(0.011)$ \\
\hline DC & $0.413[0.174 ; 0.652]$ & $1.283[1.190 ; 1.919]$ & 3.391 & $(0.0007)$ \\
\hline$\hat{\beta}_{0}$ & $0.2153[-0.2062 ; 0.6367]$ & & 1.001 & $(0.3168)$ \\
\hline$\hat{\kappa}$ & $0.4959[0.4543 ; 0.5411]$ & & & \\
\hline $\ln (v k)$ & 1 & & & \\
\hline$L(\beta)$ & 29485.34 & & & \\
\hline$D^{* p}$ & 2927.06 & & & \\
\hline$\chi^{* 2}$ & 3009.24 & & & \\
\hline $\operatorname{AIC}\left(\sigma^{2}\right)$ & 10149.43 & & & \\
\hline $\operatorname{BIC}\left(\sigma^{2}\right)$ & 10207.83 & & & \\
\hline$R_{\mathrm{D}}^{2}$ & 30.115 & & & \\
\hline
\end{tabular}

$E\left[\Lambda_{h}\right]=\Lambda_{h}$ and

$\operatorname{Var}\left[Y_{h}\right]=\Lambda_{h}+\frac{\Lambda_{h}^{2}}{\kappa}$

This model for the negative binomial distribution shows that it can be considered a more variable Poisson distribution.

In this work, negative binomial regression models with the log link function was used.

Different goodness of fit statistics were analyzed following Hardin and Hilbe (2007). Model deviance and the Pearson chisquare statistics were used as overall measures of the goodness of fit. Additionally, an intuitive measure of the explained deviations based on the likelihood ratio or deviance, $R_{\mathrm{D}}^{2}$ defined as $R_{\mathrm{D}}^{2} \%=$ $100 \times\left(1-(n-2 / n-p)\left(D^{p} / D^{0}\right)\right)$, where $n$ and $p$ are the sample size and the parameter number, respectively, $D^{0}$ is the deviance of the model with no covariates, and $D^{p}$ is the deviance of the model with $p$ parameters. Other measures, were also evaluated, such as the Akaike Information Criterion (Bozdogan, 1987) calculated as AIC = $n \log \left(\hat{\sigma}^{2}\right)+2 p$, and the Bayesian Information Criterion (Schwarz, 1978) calculated as BIC $=n \log \left(\hat{\sigma}^{2}\right)+p \log (n)$, where $\hat{\sigma}^{2}$ is the maximum likelihood estimator of the residual variance, and $n, p$ have the meanings explained above.

Wald inference based on the properties of the maximum likelihood estimators was used to test hypotheses and construct intervals on the individual model parameters.

As the models were estimated as logarithmic models, the elasticities are equivalent to the value of the coefficient estimated, and the changes in the responses associated with the percent changes in the continuous traffic variables provide a measure of their individual impacts. Incidence rate ratios IRR $=\exp (\hat{\beta})$ and $95 \%$ confidence intervals were calculated to provide additional information about the relative robustness of different policy specifications to policy makers.

Finally, the model was used to evaluate different scenarios in the transport corridors. In Section 6.1, the model adjusted for the 2001 data was used to predict accidents in the corridor Madrid-Barcelona (MB) corridor for the years 2002 and 2003, as a way to validate the model, by comparing the number of real observed accident with the predicted values for those 2 years. In Section 6.2, the model was used to simulate different hypothetical traffic scenarios.

\section{Results}

The transformed covariates $\ln (\mathrm{AADT})$, and $\ln (\% \mathrm{HGV})$, categorical variable $\operatorname{Road}_{i}$ with $i=\mathrm{AP}, \mathrm{AV}, \mathrm{DC}, C$, denoting the functional road type where $C$ is considered to be the reference road, and interaction between the traffic and categorical variables modeled by $\ln (\% \mathrm{HGV})_{i}$ were included in the final negative binomial model. The crossed effects are included to draw traffic patterns by road types, and $\ln (\mathrm{vk})$ is the parameter describing the exposure. A criteria for variables inclusion was used by testing the likelihood ratio (LR) to measure the contribution to log-likelihood. In addition, the correlation structure between them was prevented.

The equation in a segment $j$ of road type $i=\mathrm{AP}, \mathrm{AV}, \mathrm{DC}$ is

$$
\begin{aligned}
\widehat{E\left[Y_{i j}\right]}= & \exp \left(\hat{\beta}_{0}+\hat{\beta}_{1} \ln \left(\mathrm{AADT}_{j}\right)+\hat{\beta}_{2} \ln \left(\% \mathrm{HGV}_{j}\right)+\widehat{\alpha_{i}}+\widehat{\delta_{i}} \ln \left(\% \mathrm{HGV}_{j}\right)\right. \\
& \left.+\ln \left(\mathrm{vk}_{j}\right)\right)
\end{aligned}
$$

Evaluation of the model was performed by examination of the significance of the variables included using the $t$-test, in addition to engineering and intuitive judgment to confirm the validity and the practicality of the sign of each covariate since causal models with logical structure are sought. The goodness-of-fit statistics (i.e., AIC, BIC, log-likelihood, deviation, $\chi^{2}$ ), and the evaluation of the predictive and explanatory power $R_{\mathrm{D}}^{2}$ of one set of competitive models, do suggest the selected model to examine the relationship between accidents and HGVs traffic pattern by road type. The estimated overdispersion parameter $\hat{\kappa}$ is lower than 1 , indicating that overdispersion is not present if the negative binomial model is fitted.

The estimated regression and overdispersion parameters $(\hat{\beta} ; \hat{\kappa})$ determined by the maximum likelihood and the incidence rate ratio IRR $=\exp (\hat{\beta})$, along with their $95 \%$ confidence intervals, Wald statistics, and the $p$-value (in parenthesis) are displayed in Table 3. In addition, different goodness-of-fit statistics used to select the model for applications, including log-likelihood, deviation, and $\chi^{2}$, and the measure of explained deviation ( $\left.R_{\mathrm{D}}^{2} \%\right)$, as well as AIC and BIC measures are summarised in Table 3.

Globally, the effect of $\ln (\% \mathrm{HGV})$ and $\ln (\mathrm{AADT})$ decreases on all types of roads, due to the large influence of the sample of single carriageway roads on the whole. However, the type of road has an influence on the interaction with the percentage of HGVs, which indicates that there is a differentiated behaviour between single carriageways and high capacity roads. 


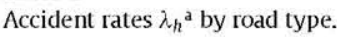

\begin{tabular}{|c|c|c|c|c|c|}
\hline & Global & $\mathrm{AP}$ & $\mathrm{AV}$ & DC & C \\
\hline$\widehat{\lambda_{h}}$ & 0.264 & 0.104 & 0.20 & 0.209 & 0.290 \\
\hline $\operatorname{IP}\left(\Lambda_{h}\right)[95 \%]$ & {$[0 ; 1.001]$} & {$[0 ; 0.396]$} & {$[0 ; 0.567]$} & {$[0 ; 0.804]$} & {$[0 ; 1.098]$} \\
\hline $\bar{\lambda}_{h}$ & 0.259 & 0.106 & 0.162 & 0.213 & 0.324 \\
\hline Differences (\%) & 2.13 & -1.60 & -7.65 & -1.92 & -10.37 \\
\hline
\end{tabular}

$\bar{\lambda}_{h}$ : observed accident rate; $\widehat{\lambda_{h}}$ : calculated accident rate by Eqs. (2). $\Lambda_{h}$ : safety at a new site. IP ( $\left.\Lambda_{h}\right)$ : prediction interval of safety at a new site.

${ }^{a}$ Rates are accidents with injured people per 1 million vehicle-kilometres $\left(A_{\mathrm{T}_{i}} / \mathrm{vk}_{\mathrm{i}}\right)$.

The crossed effect $\ln (\% \mathrm{HGV})$ and Road $_{i}$, modelled by $\ln \left(\% \mathrm{HGV}_{i}\right)$, has the opposite sign from that found between $\ln (\% \mathrm{HGV})$ and response, reversing the fundamental relationship in AP, AV, and DC road types, and determining a different behaviour of $C$ roads within the Spanish network.

The coefficients of the road type variable $\left(\operatorname{Road}_{i}\right)$ reveal significant differences (according to Pearson's $\chi^{2}$ ) between high capacity and single carriageway roads. The signs associated with high capacity roads are negative and show that the predicted number of accidents on these roads is lower than for single carriageway roads. The hypothesis test performed for the coefficients corresponding to high capacity roads reveals that there are not significant differences between them. Likewise, the hypotheses test on the coefficients corresponding to the interaction $\ln (\% \mathrm{HGV})-\operatorname{Road}_{i}$, particularized to $\mathrm{AP}$ and $\mathrm{AV}$ does not reject the null hypothesis.

The expressions of the accident rates by road types ( $i$ ) and segment $(j)$ are calculated to be

$\hat{\lambda}_{i j}=\frac{\widehat{E\left[Y_{i j}\right]}}{\mathrm{vk}_{j}}$

$\hat{\lambda}_{\mathrm{AP}_{j}}=0.2736\left(\mathrm{AADT}_{j}\right)^{-0.1111}\left(\% \mathrm{HGV}_{j}\right)^{0.0638}$

$\hat{\lambda}_{\mathrm{AV}_{j}}=0.4368\left(\mathrm{AADT}_{j}\right)^{-0.1111}\left(\% \mathrm{HGV}_{j}\right)^{0.0103}$

$\hat{\lambda}_{\mathrm{DC}_{j}}=0.3405\left(\mathrm{AADT}_{j}\right)^{-0.1111}\left(\% \mathrm{HGV}_{j}\right)^{0.2491}$

$\hat{\lambda}_{C_{j}}=1.2402\left(\mathrm{AADT}_{j}\right)^{-0.1111}\left(\% \mathrm{HGV}_{j}\right)^{-0.1639}$

\subsection{Average rates}

For all of the segments included in this work, Eqs. (2) were applied in order to calculate point estimates of the accident rate. In addition, prediction intervals were obtained according to the approach given by McCullagh and Nelder (1989) and Wood (2005).

To generalize the results, point estimates of the accident rates $\hat{\lambda}_{h}$, and the prediction interval for the safety, $\Lambda_{h}$, of a new segment of each type of road are presented in Table 4. The calculations were performed assuming that the covariate values of the new segment are the mean values of the regressors in the respective type of road. Also, in Table 4 , the average of the observed accident rates $\bar{\lambda}_{h}$ is presented.

The calculated value for $C$ roads turned out to be 0.290 , which is about 3 times the calculated value for AP roads, twice that for the $A V$ roads, and 1.5 times that corresponding to DC roads.

The calculated values show large differences between $C$ roads and the other types AP, AV, which are usually classified in the same road class. In fact, the Ministry of Public Works (MFOM) includes $\mathrm{AP}, \mathrm{DC}$ and $\mathrm{AV}$ roads in one homogeneous group denoted by the high capacity roads category, which are generally considered as inherently safer routes. However, the single carriageway roads (C) include intersections and railroad grade crossing, and the traffic flow in both directions is not separated. Therefore a higher crash risk was expected than for the unfied class, and this was observed.

When the analysis was performed by road type, the greater heterogeneity on single carriageway roads allowed establishing the hypothesis of differential behaviour and a higher accident rate than other road types, which is reflected in the previous figures.

For DC roads, an intermediate behaviour between AP, AV and $C$, can be seen, which agress with what was expected, since although there is more than one lane in both directions, as with AP and AV roads, there is no traffic flow separation as in type $C$ roads.

These results agree with the observed data. The number of accidents per vehicle-km produced in the Spanish RCE is larger for this class of roads than motorways like AP or AV.

From annual mobility and accident figures on Spanish RCE roads for the 1990-2004 period an average value for the accident rate of 0.143 is obtained on high capacity roads and 0.328 on single carriageway roads, which confirms the values found using the adjusted model.

\subsection{Elasticities and incidence rate ratios}

One of the key uses of the coefficients is the evaluation of the relative elasticities of the covariates in the accident rate. Since logarithmic models provide an straightforward way to quantify relative effects by means of estimated coefficients, their confidence intervals can also be used to bound inferences.

In this case, $\ln (\mathrm{AADT})$ is more relevant when explaining the response of $A P$ and $A V$ roads, while $\ln (\% \mathrm{HGV})$ is on DC and $C$ roads, although all parameters can be considered inelastic.

For instance, a variation in AADT could have the same impact regardless of road type, because the terms for the interaction between AADT and road type are not statiscally significant.

Different behaviour could be expected for the variation \% HGV between high capacity roads (AP, AV, and DC) and single carriageway roads, denoted by C. A $10 \%$ variation in $\% \mathrm{HGV}$, induces a $0.6 \%$ increase in AP rates, $0.1 \%$ in $\mathrm{AV}$, or $2.5 \%$ in $\mathrm{DC}$, but causes a decrease of $1.6 \%$ in $C$ roads.

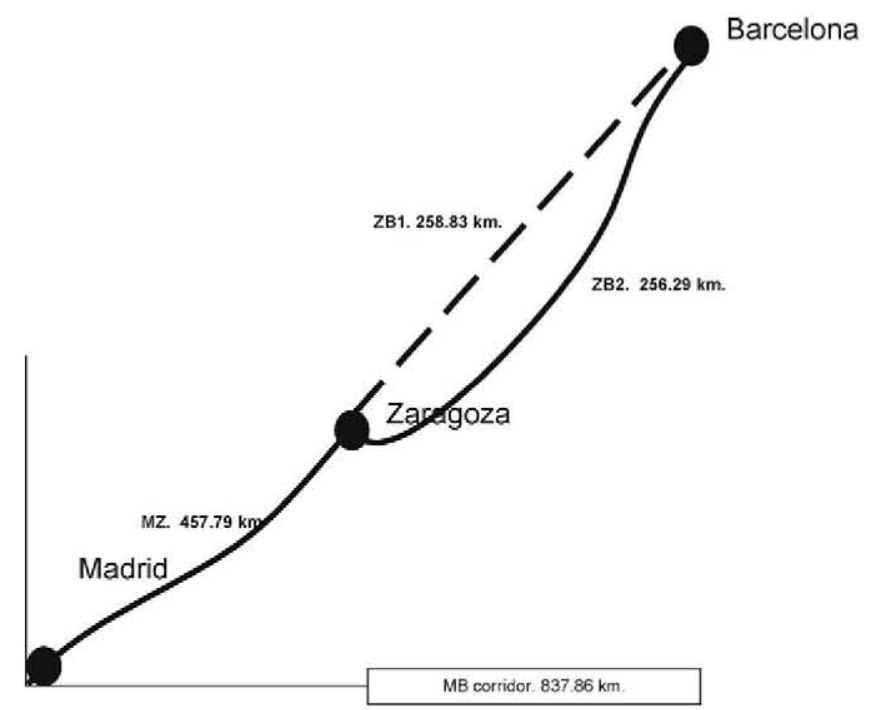

Fig. 4. Madrid Barcelona corridor (MB). 


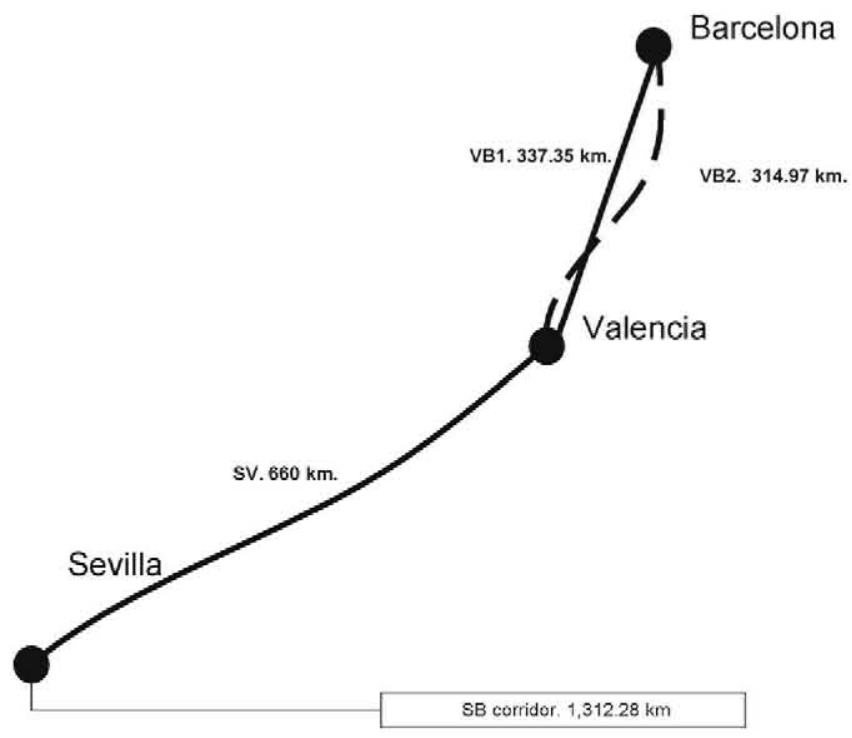

Fig. 5. Sevilla-Barcelona corridor (SB).

In addition, the incidence rate ratio IRR can be used to interpret the effect of the variables. If the IRR is much less than 1.0, an increase in the value of a variable (keeping other factors constant), is associated with an improvement of safety. For example, this could be the result of an increment of $\ln (A A D T)$ or changing one segment of a single carriageway $(C)$ with one of a motorway (AP). On the other hand, if the IRR is much greater than 1.0, a significant decline in safety could be observed. This is the case for interaction of $\ln (\% \mathrm{HGV})-$ Road $_{i}$, since an increase in \%HGV induces an increase in AP, AV, and DC accident rates, decreasing the safety performance in high capacity roads.

This kind of interpretation is relevant in that it provides a first ranking of the effects to be expected by the adoption of policies for road transport according to road type.

As a policy example, a modal shift strategy focusing on freight transport could encourage the decongestion of transport corridors by means of the incentivizing co-modality between road transport and railways, and promoting differential heavy goods vehicles reduction with consequences for road safety performance.
It may also be of help to evaluate the cost-benefit ratio of any decision to transform type $C$ segments into AV or AP segments.

The model was applied in a single approach to specific freight transport corridors, conceived as different road segments joined in an orderly pattern, which will be formally called routes.

\section{Application of the model to two important Spanish goods transport corridors}

The selected transportation corridors are Madrid-Barcelona (MB, $837.86 \mathrm{~km}$ ) and Sevilla-Barcelona (SB, $1312.38 \mathrm{~km}$ ) These corridors were chosen for two reasons: they are representative of the five most important transport corridors for Spanish goods regarding the amount of material transported, and they include segments of different road types (AP, AV, and $C$ ), while presenting alternative routes on part of the journey.

The Madrid-Barcelona corridor (Fig. 4) has a common $457.79 \mathrm{~km}$ long stretch as far as Zaragoza (MZ) that comprises 37 segments of AV. The Zaragoza-Barcelona route (ZB) has two alternatives. The first (ZB1) is $258.83 \mathrm{~km}$ long and comprises 13 AV segments (123.78 km long) and $10 \mathrm{C}$ segments (136.53 km long). The second alternative (ZB2) is made up of $21 \mathrm{AP}$ segments with a total length of $256.29 \mathrm{~km}$.

The Sevilla-Barcelona corridor (Fig. 5) has a common $660 \mathrm{~km}$ long stretch as far as Valencia (SV), comprising 40 AV segments making up $82 \%$ of the route, and $10 C$ segments making up $18 \%$ of its length.

The Valencia-Barcelona route (VB) presents two alternatives. The first (VB1), with a length of $337.35 \mathrm{~km}$, comprises $31 \mathrm{seg}$ ments of high capacity road, of which 28 segments are AP with a total length of $308.53 \mathrm{~km}$ and 3 AV segments of $28.82 \mathrm{~km}$. The second $314.97 \mathrm{~km}$ alternative (VB2) comprises 5 AV segments of $13.22 \mathrm{~km}$ and $51 \mathrm{C}$ segments which make up $95.8 \%$ of the total route length.

\subsection{Modelling accident counts in Madrid-Barcelona (MB) corridor}

The accident prediction model adjusted for the 2001 data was used to predict accidents in the corridor MB for the years 2002 and 2003, the results of which are shown in Figs. 6 and 7. For the different segments comprising the corridor, the figures show the
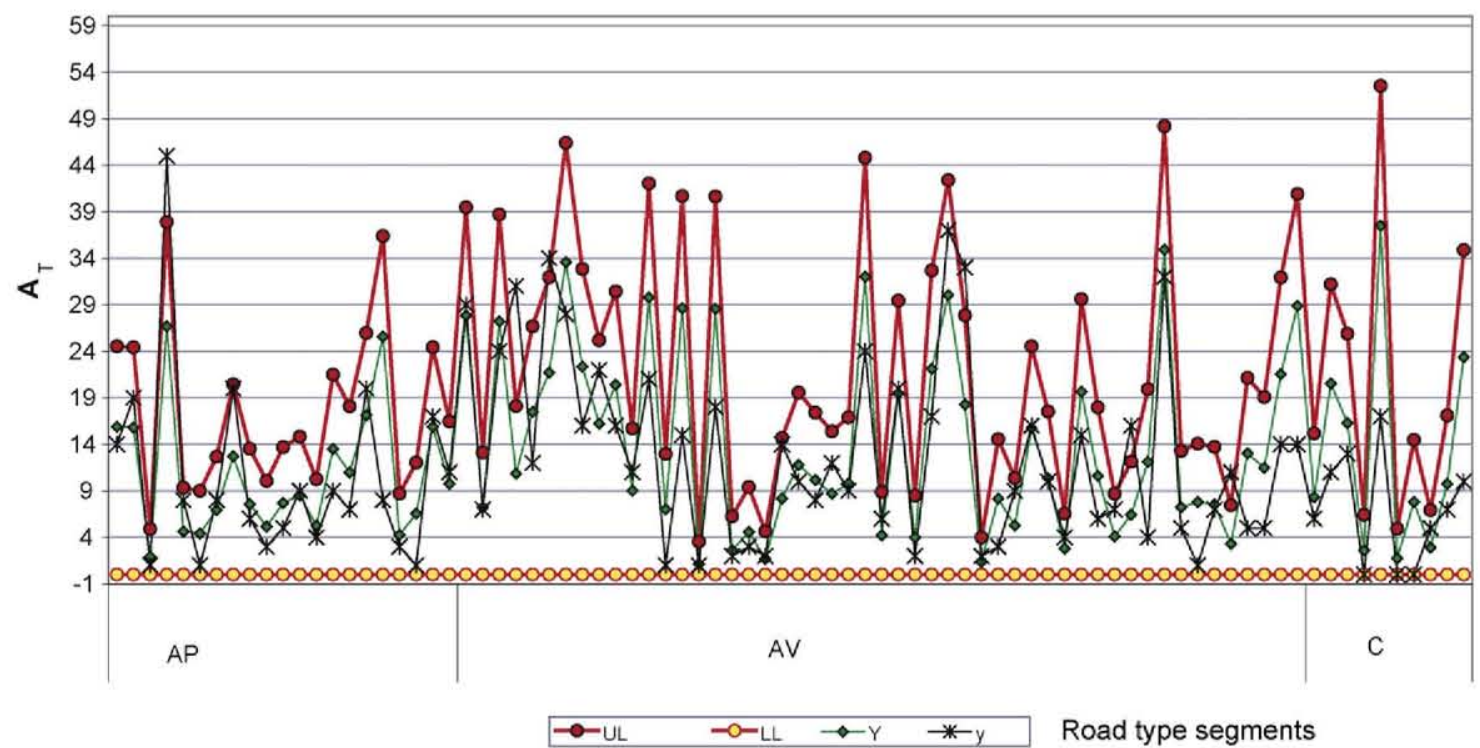

Fig. 6. Predicted $(Y)$ and observed $(y)$ accidents along MB corridor. Upper and lower prediction interval (UL-LL). Year 2002. 


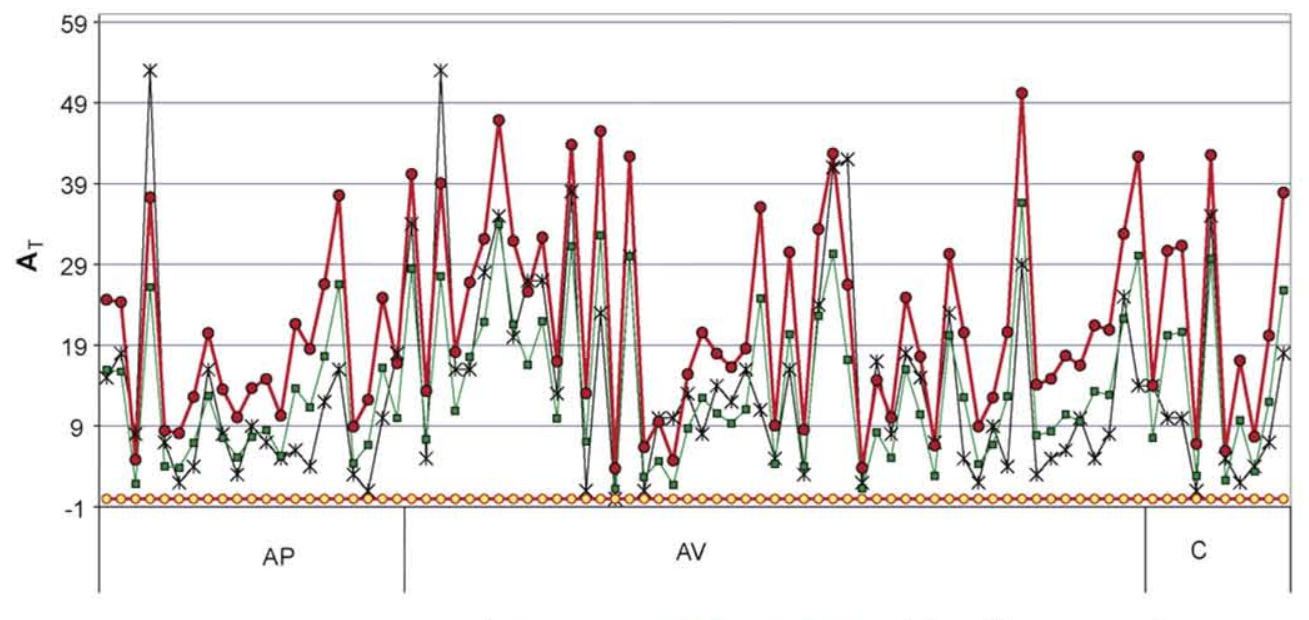

$* y \rightarrow-Y \rightarrow-U L \quad \rightarrow-L L$ Road type segments

Fig. 7. Predicted $(Y)$ and observed $(y)$ accidents along MB corridor. Upper and lower prediction interval (UL-LL). Year 2003.

number of accidents predicted by the model and the limits of the prediction interval along with the observed values. The accident figures observed during these years on the segments comprising the corridor are included in the prediction interval provided by the model, with the exception of a very small number of segments. These results show that the model can adequately predict the number of accidents on the MB corridor under existing conditions, and therefore the model is valid for use as a simulation and analysis tool for theoretical scenarios, under the assumption that the expected values of the variables do not change dramatically from 1 year to the next.

\subsection{Study scenario description}

Six different hypothetical traffic scenarios were defined over the two corridors. These scenarios were formed by combining the following hypotheses for reducing the average annual HGV daily traffic along the entire length of each route:

(A) $10 \%$ reduction in AADTHGV with the corresponding reduction of \% HGV.

(B) $20 \%$ reduction in AADTHGV with the corresponding reduction of \%HGV.

The hypotheses of total daily traffic mean intensity variation were formulated taking into account the likelihood that a reduction in the number of heavy goods vehicles could be attractive to other users and produce induced traffic. For this, three possible situations were considered:

(a) $10 \%$ reduction (or $20 \%$ ) in AADT with the corresponding reduction of vk.

(b) $0 \%$ change in AADT with the 2001 level of vk exposure maintained by other vehicular traffic replacing the number of HGVs subtracted.

(c) $10 \%$ increase (or $20 \%$ ) in AADT, by the addition of other traffic, with the corresponding increase in vk exposure.

The combination of these hypotheses gives rise to six scenarios that can be summarized in Table 5 .

\subsection{Study scenario results}

The results of applying Eq. (1) and the different scenario hypotheses to the sections of each road type in the MB and SB corridors are shown in Tables 6-9, in terms of percentage variation in mobility (vk) and the number of accidents $\left(A_{T}\right)$ with respect to the 2001 data. For example, in Table 6 for scenario $A a$, the $10 \%$ traffic reduction in HGVs, without other induced vehicle traffic induction would cause a $0.81 \%$ reduction in total mobility and a $15.43 \%$ reduction in the total number of accidents over the whole of the AP stretches of the MB corridor.

From the results obtained, the following significant facts may be deduced: for all the scenarios on the high capacity roads (AP and $\mathrm{AV}$ ) there is a significant reduction in the total number of accidents, which varies between $16.38 \%$ for the Ba scenario of the MB corridor and $7.35 \%$ for the Bc scenario of the SB corridor. Likewise, on the sections of single carriageway roads $(C)$ there are significant increases in the total number of traffic accidents, with a maximum increase of $28.06 \%$ for the scenario $B c$ on the MB corridor and minimum increase of $6.52 \%$ for the scenario $A a$ on the SB corridor.

With these results, the expected reduction in the total number of accidents has been calculated globally on each of the corridors (considering the common stretch and the alternatives) and on the sections of road types comprising each corridor for each scenario. The results are shown in Figs. 8-11.

The data shown in Figs. 8-11 can be analyzed along with those given in the corresponding Tables 6-9. From Fig. 8 and Table 6, it can be concluded that for scenario $A a$ on the single carriageway roads (C), there would be a total reduction in mobility of $2.59 \%$ and an additional 19 accidents, while on the AP segments, mobility would be reduced by $0.81 \%$ with a 40 fewer accidents. On AV sections,

Table 5

Scenarios of AADTHGV changes with and without any change to total vehicular traffic intensity (AADT).

\begin{tabular}{|c|c|c|c|}
\hline & \multicolumn{3}{|c|}{$A A D T$ changes } \\
\hline$A A D T H G V$ changes & a: $\left\{\begin{array}{c}-10 \% \\
\text { o } \\
-20 \%\end{array}\right.$ & $\mathrm{b}: 0 \%$ & $\mathrm{c}:\left\{\begin{array}{c}10 \% \\
\mathrm{o} \\
20 \%\end{array}\right.$ \\
\hline$A:-10 \%$ & $A a$ & $A b$ & $A c$ \\
\hline B : $-20 \%$ & $B a$ & $B b$ & $B c$ \\
\hline
\end{tabular}


Table 6

Variation in vk exposure and the number of accidents in the $\mathrm{Aa}, \mathrm{Ab}$, and Ac scenarios $(\Lambda \mathrm{i}, \mathrm{i}=\mathrm{a}, \mathrm{b}, \mathrm{c})$ over the MB corridor $\left(\Delta \mathrm{vk} \% ;\left(\Delta A_{\mathrm{T}} \%\right)\right.$ ).

\begin{tabular}{llll}
\hline Road type & Aa & Ab & AC \\
\hline AP & $-0.81 \%(-15.43 \%)$ & $0 \%(-14.79 \%)$ & \\
AV & $-1.05 \%(-13.58 \%)$ & $0 \%(-12.72 \%)$ & $1.05 \%(-11.86 \%)$ \\
C & $-2.59 \%(18.18 \%)$ & $0 \%(20.96 \%)$ & $2.59 \%(23.72 \%)$ \\
MB corridor & $-1.09 \%(-11.20 \%)$ & $0 \%(-10.22 \%)$ & $1.09 \%(-9.24 \%)$ \\
\hline
\end{tabular}

Table 7

Variation in vk exposure and the number of accidents in the $\mathrm{Ba}, \mathrm{Bb}$, and $\mathrm{Bc}$ scenarios (Bi, $\mathrm{i}=\mathrm{a}, \mathrm{b}, \mathrm{c})$, over the $M B$ corridor $\left(\Delta \mathrm{vk} \% ;\left(\Delta \mathrm{A}_{\mathrm{T}} \%\right)\right.$ ).

\begin{tabular}{llll}
\hline Road type & $\mathrm{Ba}$ & $\mathrm{Bb}$ & $\mathrm{Bc}$ \\
\hline $\mathrm{AP}$ & $-1.61 \%(-16.38 \%)$ & $0 \%(-15.11 \%)$ & $1.61 \%(-13.85 \%)$ \\
$\mathrm{AV}$ & $-2.11 \%(-14.48 \%)$ & $0 \%(-12.76 \%)$ & $2.11 \%(-11.04 \%)$ \\
$\mathrm{C}$ & $-5.17 \%(16.83 \%)$ & $0 \%(22.46 \%)$ & $5.17 \%(28.06 \%)$ \\
MB corridor & $-2.18 \%(-12.15 \%)$ & $0 \%(-10.19 \%)$ & $2.18 \%(-8.23 \%)$ \\
\hline
\end{tabular}

reductions in mobility and the number of accidents would be $1.05 \%$ and 108, respectively. For the same scenario on the MB corridor, the global result would be a $1.09 \%$ decrease in exposure and 130 fewer accidents.

By analyzing the overall set of results shown in Figs. 8-11 (and corresponding Tables 6-9), for the two corridors analyzed and the six scenarios defined, the following may be highlighted:

- In all cases, a reduction in the total number of accidents would occur as a result in the drop in the number of heavy goods transport vehicles (AADTHGV). This drop varies between 46 for the SB corridor Bc scenario and 141 for the MB corridor Aa scenario. These figures were obtained because the expected reductions on high capacity roads (AP and AV) would exceed the increases on the sections of single carriageway road $(C)$. On corridors with a higher proportion of type $C$ sections, the results of could be less satisfactory, and there might even be an increase in accidents. However, the lower reduction in accidents on the SB corridor is a result of this.

- In all cases, the higher the traffic intensity (AADT) as a result of the induced vehicular traffic, the higher the number of accidents due to the increase in exposure. This effect is greater on single carriageway road segments compared to high capacity roads. In addition, the reduction in the number of HGVs is greater in the $B$ hypothesis compared to the $A$ one. For the SB route the increase in the number of accidents between scenarios $A a$ and Ac on type $C$ segments is almost $20 \%$ and between scenarios $\mathrm{Ba}$ and $\mathrm{Bc}$, it is $42 \%$. For type AP segments, the increases are $8 \%$ and $19 \%$, and for

Table 8

Variation in vk exposure and the number of accidents in the $A a, A b$, and Ac scenarios $(\mathrm{Ai}, \mathrm{i}=\mathrm{a}, \mathrm{b}, \mathrm{c})$ over the SB corridor $\left(\Delta \mathrm{vk} \% ;\left(\Delta A_{\mathrm{T}} \%\right)\right)$.

\begin{tabular}{llll}
\hline Road type & Aa & Ab & Ac \\
\hline AP & $-0.59 \%(-9.86 \%)$ & $0 \%(-9.38 \%)$ & $0.59 \%(-8.89 \%)$ \\
AV & $-0.77 \%(-8.64 \%)$ & $0 \%(-8.27 \%)$ & $0.77 \%(-7.79 \%)$ \\
C & $-0.85 \%(6.52 \%)$ & $0 \%(7.26 \%)$ & $0.85 \%(7.85 \%)$ \\
SB corridor & $-0.73 \%(-4.36 \%)$ & $0 \%(-3.85 \%)$ & $0.73 \%(-3.34 \%)$ \\
\hline
\end{tabular}

Table 9

Variation in vk exposure and the number of accidents in the $\mathrm{Ba}, \mathrm{Bb}$, and $\mathrm{Bc}$ scenarios (Bi, i=a, b, c) over the SB corridor $\left(\Delta \mathrm{vk} \% ;\left(\Delta A_{\mathrm{T}} \%\right)\right.$ ).

\begin{tabular}{llll}
\hline Road type & $\mathrm{Ba}$ & $\mathrm{Bb}$ & $\mathrm{BC}$ \\
\hline $\mathrm{AP}$ & $-1.18 \%(-10.53 \%)$ & $0 \%(-9.56 \%)$ & $1.18 \%(-8.60 \%)$ \\
AV & $-1.55 \%(-9.04 \%)$ & $0 \%(-8.31 \%)$ & $1.55 \%(-7.35 \%)$ \\
$\mathrm{C}$ & $-1.69 \%(6.42 \%)$ & $0 \%(7.91 \%)$ & $1.69 \%(9.09 \%)$ \\
SB corridor & $-1.46 \%(-4.73 \%)$ & $0 \%(-3.71 \%)$ & $1.46 \%(-2.69 \%)$ \\
\hline
\end{tabular}

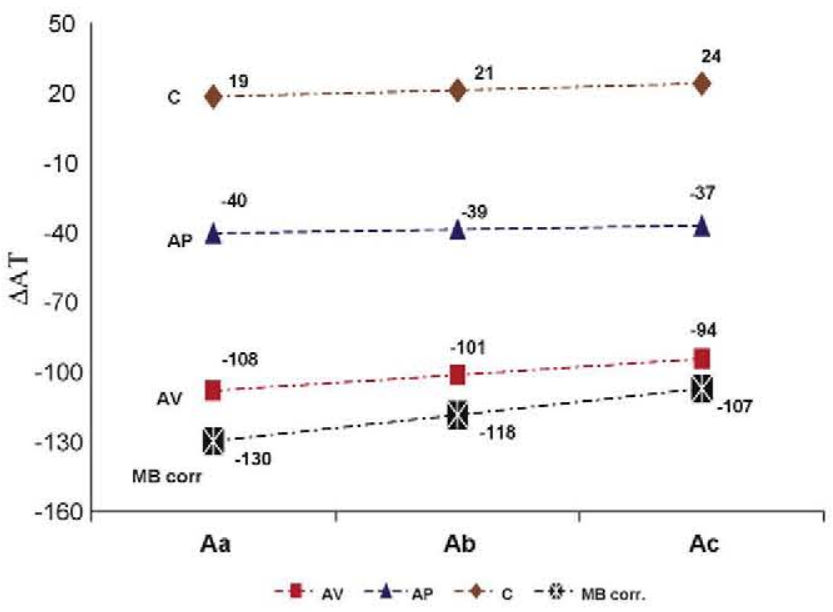

Fig. 8. Variation in the number of accidents $\left(\Delta A_{\mathrm{T}}\right)$ on the $\mathrm{MB}$ corridor for the $\mathrm{Aa}$, $\mathrm{Ab}$, and $\mathrm{Ac}$ scenarios. 2001 data in Spain.

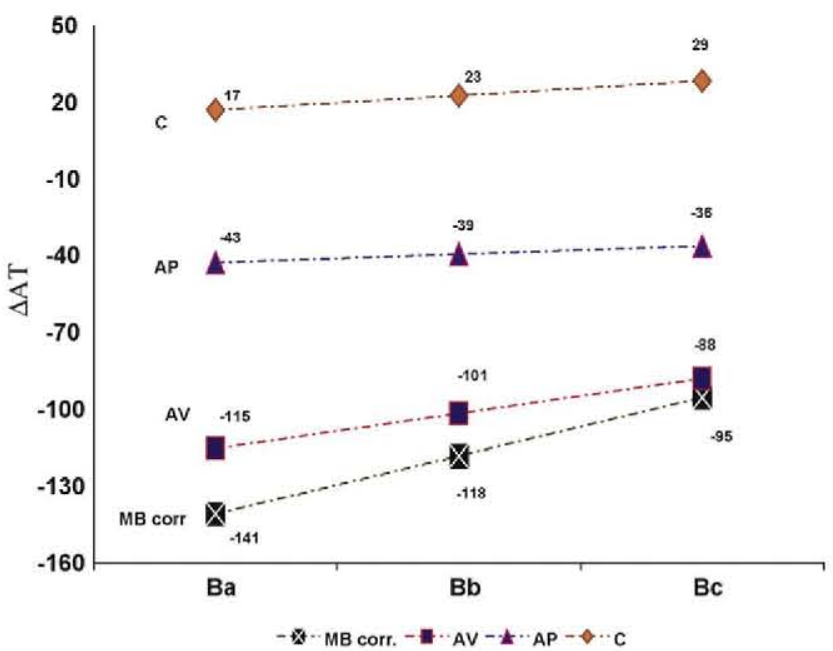

Fig. 9. Variation in the number of accidents $\left(\Delta A_{T}\right)$ on the $M B$ corridor for the $B a$, $\mathrm{Bb}$, and $\mathrm{Bc}$ scenarios. 2001 data in Spain.

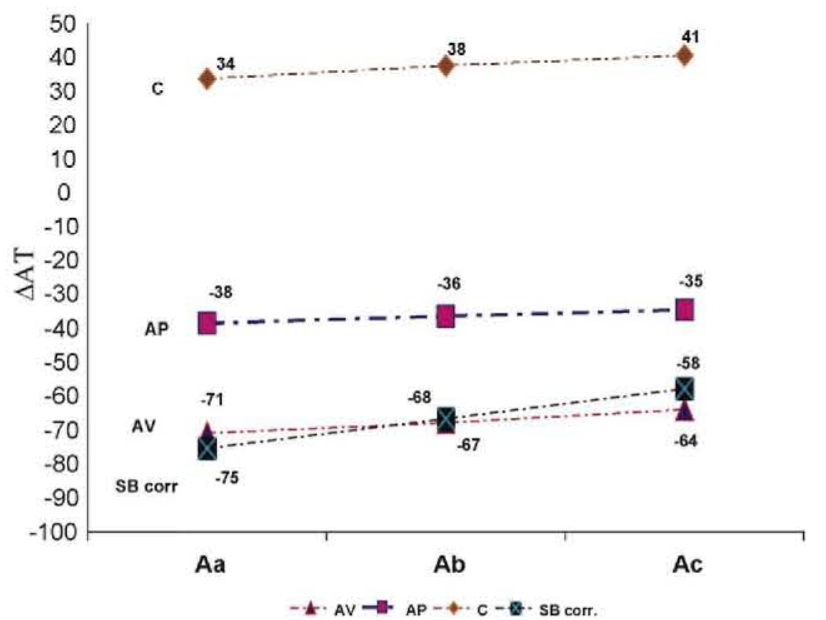

Fig. 10. Variation in the total number of accidents $\left(\Delta A_{T}\right)$ on the SB corridor for the $\mathrm{Aa}, \mathrm{Ab}$, and Ac scenarios. 2001 data in Spain. 


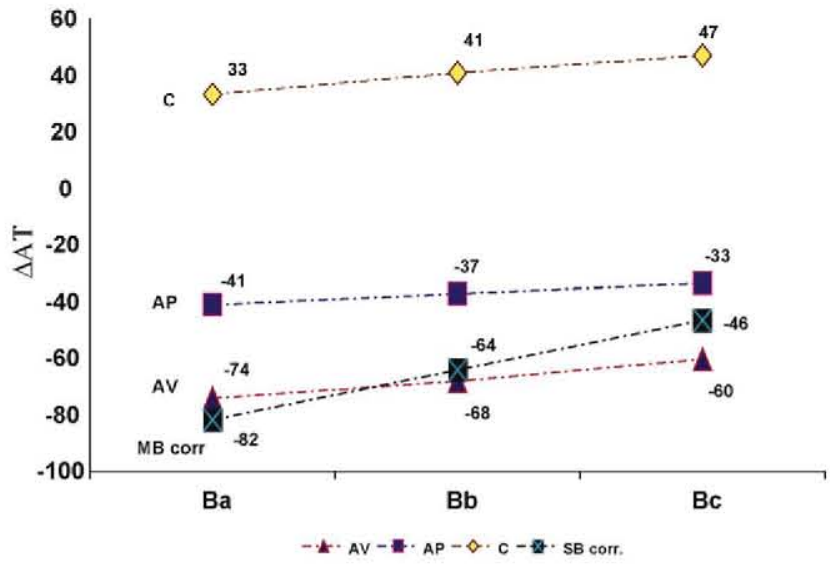

Fig. 11. Variation in the number of accidents $\left(\Delta A_{\mathrm{T}}\right)$ on the SB corridor for the $\mathrm{Ba}$, $\mathrm{Bb}$, and Bc scenarios. 2001 data in Spain.

type AV segments the increases are $10 \%$ and $19 \%$, respectively. For the corridor, the variation in the total number of accidents from scenario $\mathrm{Aa}$ to $\mathrm{Ac}$ is a $23 \%$ increase, and when changing from $\mathrm{Ba}$ to $\mathrm{Bc}$ is a $43 \%$ increase.

\section{Conclusions}

- A methodology was developed for analyzing the influence of the percentage of heavy goods transport vehicles and the total traffic on accidents on different types of interurban roads, by applying negative binomial regression models. The results obtained agree with what was expected.

- The model can adequately predict the number of accidents on specific corridors, under existing conditions for several years, at least, in the short term, and therefore validate the model for use as a simulation and analysis tool for theoretical scenarios.

- The developed model was applied to two of the most important traffic corridors in Spain: Madrid-Barcelona and Sevilla-Barcelona, comprising sections of different road types (AP, $\mathrm{AV}$, and $C$ ), with two alternative routes on parts of the main routes. Six hypothetical scenarios were also considered, including reductions of 10 and $20 \%$ in heavy goods vehicle traffic for each corridor, combined with reducing, maintaining, or increasing exposure (vk) as a result of new induced traffic due to a reduction in heavy goods vehicle traffic.

- In all the scenarios, there are reductions in the total number of accidents on sections of high capacity road (AV and AP), while there is an increase on sections of single carriageway roads $(C)$. The net effect on both routes is a reduction in the number of accidents due to the fact that the road network has relatively few single carriageway roads $(C)$, and the increase in accidents on these is compensated for, and exceeded by, reductions on high capacity sections of roads (AV and AP).

- The increase in exposure as a result of induced vehicular traffic due to a drop in the number of HGVs has a negative effect on safety, which is converted into a rapid increase in the total number of accidents on the segments of single carriageway roads $(C)$ and in a lower reduction on the AP and AV segments. This deterioration in safety may be explained by the greater influence of light vehicles (mainly cars) on accidents, compared to the influence of heavy goods vehicles with similar mobility. Another reason for this behaviour could be that the increase in other vehicular traffic could produce an increase in the difference in speed between the slowest and the fastest vehicles, and contribute to the decrease of safety performance, as the results published by other researchers have shown. In addition, on single carriageway roads, the increase in light vehicles traffic could produce scenarios of even greater traffic conflicts caused by overtaking maneuvres, using the oncoming lane, thereby increasing the risk of accidents.

The following may be stated as more general conclusions:

- The model was found to be a useful tool for evaluating the effects of modal transfer policies between roads and rails or other forms of transport.

- It may also be of help to evaluate the cost-benefit ratios of any decisions to transform type $C$ segments into AV or AP segments.

- Applying the model to specific routes in order to analyze any possible influence of heavy goods traffic on the total number of accidents gives results which agree with those that are expected.

- It would be useful carry out studies where a greater number of routes are analyzed with different layouts and scenarios, with the purpose of better setting the limits for applying the model and methodology used.

- It is also recommended to study the effect of other types of variables, including speed and speed differential measures, as well as variables that better qualify the road type (such as geometry and route layout) which will help to clarify the behaviours detected.

\section{Acknowledgements}

The work described in this paper was funded by a grant from the Ministry of Public Works (MFOM) and was conducted at the Automobile Research Institute (INSIA) of the Polytechnic University of Madrid (UPM), during 2004-2005. Camino González work was also partially supported by Spanish Ministry of Education, Culture and Sport (MEC) under contract MTM 2005-08897. The authors thanks the two anonymous referees for their comments which have greatly contributed to the improvement of the work, and to J. Ollier for the translation of the manuscript into English.

\section{Appendix A}

- Variables

- AADT: annual average daily traffic

- AADTHGV: annual average daily traffic of heavy goods vehicles

- $A_{T_{i}}$ : accident counts in each road segment

- AP: toll motorways

- AV: roads with limited access and free motorways

$C$ : single carriageway roads

DC: two undivided dual carriageway roads

$\% \Delta A_{T_{i}}$ : percentage increase/decrease in number of accidents

$0 \% \Delta v \mathrm{k}$ : percentage increase/decrease of vehicle-kilometres

- HGV: heavy gross vehicles, heavy goods vehicles

\%HGV: percentage of heavy goods vehicles of annual average daily traffic

$0 \lambda$ : accident rate, accidents with injured people per 1 million vehicle-kilometres.

o vk: exposure measure defined as kilometres travelled by vehicles along RCE road segments.

- Abbreviations

- DGT: General Directorate of Road Traffic (Dirección General de Tráfico)

- ETSII: Higher Technical School of Industrial Engineering. (Escuela Técnica Superior de Ingenieros Industriales)

- INSIA: Automobile Research Institute (Instituto Universitario del Automóvil) 
- MEC: Ministry of Education, Culture and Sports (Ministerio de Educación, Cultura y Deporte)

- MFOM: Ministry of Public Work (Ministerio de Fomento)

- RCE: Spanish Interurban Road State Network (Red de Carreteras del Estado)

- UPM: Polytechnic University of Madrid (Universidad Politécnica de Madrid)

- PESV05-08: 2005-2008 Strategic Road Safety Plan (Plan Estratégico de Seguridad Vial, 2005-2008)

- MB: Madrid-Barcelona corridor

- MZ: Madrid-Zaragoza route

- ZB: Zaragoza-Barcelona ruote

- SB: Sevilla-Barcelona corridor

- SV: Sevilla-Valencia route

- VB: Valencia-Barcelona route

\section{References}

Abdel-Aty, M.A., Radwan, A.E., 2000. Modeling traffic accident occurrence and involvement. Accident Analysis and Prevention 32 (5), 633-642.

Accidents database, 2001. Dirección General de Tráfico, Spain.

MFOM, 2005. Los transportes y los servicios postales. Informe anual 2005. Ministerio de Fomento, Spain.

Bozdogan, H., 1987. Model selection and Akaike's Information Criterion (AIC): the general theory and its analytical extensions. Psychometrika 52, 345370.

PESV05-08, 2005. Plan Estratégico de Seguridad Vial 2005-2008. Dirección General de Tráfico. Spain.

Fridstrøm, L., Ifver, J., Ingebrigtsen, S., Kulmala, R., Thomsen, L., 1995. Measuring the contribution of randomness, exposure, weather, and daylight to the variation in road accident counts. Accident Analysis and Prevention 27 (1), 1-20.

Garber, N., Wu, L., 2001. Stochastic models relating crash probabilities with geometrics and corresponding traffic characteristics data. Research Report
No. UVACTS-5-15-74. Center for Transportation Studies at the University of Virginia.

Hardin, J.W., Hilbe, J.M., 2007. Generalized Linear Models and Extensions, 2nd ed. Stata Press.

Hiselius, L.W., 2004. Estimating the relationship between accident frequency and homogeneous and inhomogeneous traffic flows. Accident Analysis and Prevention 36, 985-992.

Ivan, J., O'Mara, P., 1997, Prediction of traffic accident rates using Poisson regression. 76th Annual Meeting of the Transportation Research Board. Washington, D.C. Paper No. 970861.

Ivan, J.N., Pasupathy, R.K., Ossenbruggen, P.J., 1999. Differences in causality factors for single and multi-vehicle crashes on two-lane roads. Accident Analysis and Prevention 31 (6), 695-704.

Jovanis, P., Chang, H.L., 1986. Modeling the relationship of accidents to miles trav elled. Transportation Research Board 1068, 42-51.

Lardelli-Claret, P., Luna-Del-Castillo, J. Jiménez-Moleón, J., Rueda-Domínguez, T. García-Martín, M., Femia-Marzo, P., Bueno-Cavanillas, A., 2003. Association of main driver-dependent risk factors with the risk of causing a vehicle collision in Spain, 1990-1999. Annals of Epidemiology 14 (7), 509-517.

Martin, J.L., 2002. Relationship between rate and hourly traffic flow on interurban motorways. Accident Analysis and Prevention 34, 619-629.

McCullagh, P., Nelder, J.A., 1989. Generalized Linear Models, 2nd ed. Chapman \& Hall/CRC, Florida.

Miaou, S.P., Lum, H., 1993. Modeling vehicle accident and highway geometric design relationships, Accident Analysis and Prevention 25 (6), 689-709.

Shankar, V., Mannering, F., Barfield, W., 1995. Effect of roadway geomet rics and envi ronmental factors on rural freeway accidents frequencies. Accident Analysis and Prevention 27 (3), 371-389.

Schwarz, G., 1978. Estimating the dimension of a model. The Annals of Statistics 6, 461-464.

Traffic Map, 2001. Ministerio de Fomento. Dirección General de Carreteras, Madrid, Spain.

Vogt A., Bared J., 1998. Accident models for two-lane rural segments and intersections, Transportation Research Record 1635, Transportation Research Board. Washington. D.C. $18-29$.

Wood, $G, R ., 2005$. Confidence and prediction intervals for generalised linear accident models. Accident Analysis and Prevention 37, 267-273. 